\title{
The PTEN-AKT-mTOR/RICTOR Pathway in Nasal Natural Killer Cell Lymphoma Is Activated by miR-494-3p via PTEN But Inhibited by miR-142-3p via RICTOR
}

\author{
Hao-Hui Chen, Wei-Ting Huang, Li-Wei Yang, and Chung-Wu Lin
}

From The Tissue Bank, Center for Genomic Medicine, and the Graduate Institute of Pathology, College of Medicine, National Taiwan University, Taipei, Taiwan

\author{
Accepted for publication \\ January 29, 2015 \\ Address correspondence to \\ Chung-Wu Lin, M.D., Ph.D., \\ Graduate Institute of Pathol- \\ ogy, National Taiwan Univer- \\ sity College of Medicine, 1-1 \\ Jen-Ai Rd, Taipei 100, \\ Taiwan. E-mail: chungwulin@ \\ ntu.edu.tw.
}

\begin{abstract}
Nasal natural killer (NK) cell lymphoma (NNL) is an Epstein-Barr virus-associated lymphoma of cytotoxic NK cell origin. The Epstein-Barr virus-encoded miR-BART20-5p inhibits T-bet (TBX21), the master transcription factor of cytotoxic NK cells. To further explore the roles of miRNAs in NNLs, we measured the miRNA expression profiles of 36 NNLs. miR-21, miR-142-3p, miR-126, miR-451, and miR-494-3p were the top five miRNAs with the highest expression levels. By using pathway analysis, we identified associations between all of the five miRNAs with the PTEN-AKT-mTOR pathway, in which PTEN suppresses the oncogenic AKT, and mTOR mediates the oncogenic effects of AKT. YT and NK92 cells derived from NK cell lymphomas were used. miR-494-3p inhibited PTEN with secondary activation of AKT in NK92 cells, and miR-142-3p inhibited RICTOR, a key component of the mTOR complex, with secondary suppression of AKT in YT cells. Significantly, T-bet inhibited the PTEN-AKT-mTOR/RICTOR pathway through induction of PTEN and suppression of RICTOR. Therefore, a molecular circuit of T-bet, PTEN, AKT, and RICTOR is regulated by miR-BART20-5p, miR-494-3p, and miR-142-3p. This circuit is involved in the pathogenesis of NNL. Hence, antagomirs to miR-BART20-5p or miR-494-3p, miR-142-3p mimics, or AKT inhibitors may be useful in NNL therapy. (Am J Pathol 2015, 185: 1487-1499; http:// dx.doi.org/10.1016/j.ajpath.2015.01.025)
\end{abstract}

Nasal natural killer (NK) cell lymphoma (NNL) is an EpsteinBarr virus (EBV)-associated lymphoma derived from cytotoxic NK cells of the nasal mucosa. Because cytotoxic NK cells normally eradicate viral infections, it is interesting to investigate how the EBV may evade and transform NK cells in NNL.,2 Interferon (IFN)- $\gamma$ is the primary cytotoxic cytokine of NK cells, and T-bet (T-box 21/T-box expressed in T cells; official name TBX21) is the master transcription factor for IFN- $\gamma$. The EBV-encoded miR-BART20-5p inhibits both Tbet and IFNG in NNL. ${ }^{1,2}$ This inhibition allows the survival of EBV inside NK cells and possibly also the malignant transformation of NK cells. The EBV encodes at least 44 miRNAs; the functions of most EBV-encoded miRNAs in the pathogenesis of NNL remain to be explored. In addition, it is unclear how miRNAs encoded in the human genome contribute to the pathogenesis of NNL through interactions with the EBV-encoded miRNAs.
The miRNA expression profiles of normal NK cells are well known. Although the human genome encodes $>1000$ miRNAs, human CD56 ${ }^{+} \mathrm{NK}$ cells express only 48 miRNAs. Similarly, murine NK cells express only a limited set of miRNAs. In murine NK cells, the top 10 miRNAs constitute $65 \%$ of all miRNA transcripts, and miR-21 alone, the most abundantly expressed miRNA, accounts for approximately $30 \%$ of all miRNA transcripts. ${ }^{4}$

In contrast to normal $\mathrm{NK}$ cells, data on the miRNA expression profiles of malignant NK cells are limited. Both NNLs and EBV-negative peripheral T-cell lymphomas

Supported by National Science Council grants 101-2320-B-002-014 and 102-2320-B-002-011 and National Taiwan University Hospital grant NTUH-103-S2415 (both to C.W.L.).

Disclosures: None declared. 
express high levels of miR-21, but the level of miR-142-3p is lower in NNLs than in EBV-negative peripheral T-cell lymphomas. ${ }^{5}$ Furthermore, aberrant overexpression of miR-21 and miR-155 activates AKT signaling via downregulation of the tumor-suppressor PTEN in NK cell lymphoma/leukemia. ${ }^{6-8}$ Together, these data support a PTEN-AKT pathway regulated by miRNAs in NNL.

$A K T 1$ is a well-characterized oncogene, ${ }^{9}$ whose oncogenic activities are modulated by the tumor suppressors TP53 and PTEN, which are regarded as the guardian of the genome. ${ }^{10}$ There are multiple interactions among the oncogenic AKT and the tumor suppressors TP53 and PTEN. For example, TP53 increases transcription of PTEN. ${ }^{11}$ Conversely, PTEN induces TP53 via phosphatase-dependent and phosphataseindependent mechanisms. ${ }^{12}$ In the phosphatase-dependent pathway, cytokines and antigen receptor activation promote phosphatidylinositol 3,4,5 triphosphates to produce phosphorylated AKT (pAKT), which may phosphorylate mouse double minute 2 homolog to accelerate TP53 degradation. PTEN removes the phosphate from phosphatidylinositol 3,4,5 triphosphates, prevents formation of pAKT, and blocks degradation of TP53. ${ }^{13}$ In the phosphatase-independent pathway, interactions between PTEN and TP53 maintain high acetylation of TP53 and enhance the DNA binding ability of TP53. ${ }^{14}$ Through these mutual interactions, increased TP53 and PTEN may cause degradation of pAKT. ${ }^{15}$

The oncogenic effects of AKT are mediated through the mammalian target of rapamycin (mTOR), including mTOR complexes (mTORCs) 1 and 2. The mTORCs are activated by pAKT. Conversely, a key component of mTORC2, RPTORindependent companion of mTOR complex 2 (RICTOR), may phosphorylate and activate AKT. ${ }^{16}$

Both mTORC1 and mTORC2 have been implicated in the development of thymocytes and lymphoblastic lymphoma of immature T-cell origin. ${ }^{17-19}$ Furthermore, in diffuse large B-cell lymphoma, the activity of mTORC2 is important in the development of drug resistance to the mTOR inhibitor rapamycin. ${ }^{20}$ These data support an oncogenic AKT-mTOR pathway. However, regulation of the AKT-mTOR pathway by miRNAs in NNLs is not well documented.

In this study, genome-wide miRNA expression profiles in 36 NNLs showed that miR-494-3p and miR-142-3p were abundantly expressed in NNLs. A PTEN-AKT-mTOR/ RICTOR pathway was proposed, in which inhibition of RICTOR by miR-142-3p inactivates the oncogenic AKT pathway, and inhibition of PTEN by miR-494-3p activates the oncogenic AKT pathway. Finally, we showed that T-bet, the master transcription factor of cytotoxic NK cells, may regulate the PTEN-AKT-mTOR/RICTOR pathway in NNLs.

\section{Materials and Methods}

\section{Tissue Samples}

From the archives at the Pathology Department of National Taiwan University Hospital (Taipei), a series of 36 NNLs were selected. Diagnoses were made on pretreatment nasal biopsy specimens by a combination of histopathology, diffuse positive immunostaining for $\mathrm{CD} 3$, focal to diffuse positive immunostaining for $C D 56$, and in situ hybridization for EBV-encoded small RNA. ${ }^{1,2}$ The ethics committee of the National Taiwan University Hospital approved the study.

\section{Genome-Wide miRNA Profiles in 36 NNLs}

A series of 36 NNLs were used. The nCounter miRNA Expression Analysis System (NanoString Technologies, Seattle, WA) was used to obtain the expression profiles of 651 human miRNAs. ${ }^{21-23}$ A panel of six synthetic miRNAs was used as internal positive controls. Approximately $0.1 \mu \mathrm{g}$ of total RNAs was extracted from formalin-fixed, paraffinembedded tissue blocks. Mature miRNAs were ligated to sequence-specific miRtags, and the miRtagged mature miRNAs were hybridized to fluorescent reporter probes and to capture probes. After hybridization, the samples were immobilized to a solid-phase cartridge, and a chargecoupled device digital analyzer was used for data acquisition. The data were normalized to the internal positive controls (Supplemental Table S1).

\section{Cell Lines and Transfection}

YT and NK92 cells are EBV-infected lymphoma cell lines of NK cell origin. They were obtained from the German Collection of Microorganisms and Cell Culture (Braunschweig, Germany). YT cells were grown in $80 \%$ Iscove's Modified Dulbecco's Medium plus 20\% fetal bovine serum. NK92 cells were grown in $75 \% \alpha$-minimal essential medium, $12.5 \%$ fetal bovine serum, $12.5 \%$ horse serum, $2 \mathrm{mmol} / \mathrm{L} \mathrm{L}$-glutamate, and $10 \mathrm{ng} / \mathrm{mL}$ IL-2.

For transfection experiments, 20 to $40 \mu \mathrm{g}$ of enhanced green fluorescent protein-expressing plasmids was electroporated into the cells, followed by selection with G418 until $>90 \%$ were positive for enhanced green fluorescent protein.

\section{Luciferase Activity Assay for Inhibition of PTEN by miR-494-3p or RICTOR by miR-142-3p}

Luciferase assays were performed in YT or NK92 cells with a plasmid miRCS-GLO-targetCS (Supplemental Figure S1 and Table 1) derived from the pmirGLO Dual-Luciferase miRNA target expression vector (Promega, Madison, WI). The plasmid, miRCS-GLO-targetCS, includes cloning sites for both miRNA and $3^{\prime}$ untranslated region (UTR) of the target. The firefly luciferase was the primary reporter to monitor inhibition of the targets by miRNAs. A Renilla luciferase was used as an internal control for normalization.

For inhibition of PTEN by miR-494-3p, the $3^{\prime}$ UTR of $P T E N$, approximately $3.3 \mathrm{~K}$, was inserted downstream of the firefly luciferase coding region, and the wild-type (WT) or mutant (MT) miR-494 stem-loop precursor was inserted into the miRNA expression cassette. The resultant vector, 
WT-miR-494-GLO-PTEN-3'UTR, MT-miR-494-GLO-PTEN$3^{\prime} \mathrm{UTR}$, or miRCS-GLO-PTEN-3'UTR, was transfected into NK92 cells. For inhibition of RICTOR by miR-142-3p, the $3^{\prime} \mathrm{UTR}$ of RICTOR, approximately $4.4 \mathrm{~K}$, was inserted downstream the firefly luciferase coding region, and the WT or MT miR-142 stem-loop precursor was inserted into the miRNA expression cassette. The resultant vector, WT-miR-142-GLO-RICTOR-3'UTR, MT-miR-142-GLORICTOR-3'UTR, or miR-CS-GLO-RICTOR-3'UTR, was transfected into YT cells.

Luciferase activities were measured according to the manufacturer's protocol. The ratio of firefly luciferase activity/Renilla luciferase activity was obtained and was normalized to the ratio obtained when an empty vector expressing no miRNAs was used as a control.

\section{Plasmids for Localization of Target Sites with Luciferase Assay}

Two plasmids were derived from the plasmid, WT-miR-494GLO-PTEN-3'UTR, to localize the miR-494-3p binding on the $P T E N-3^{\prime}$ UTR (Supplemental Figure S1 and Table 1). In the first plasmid, WT-miR-494-GLO-MT1-PTEN-3'UTR, the seed region of the first predicted binding site at $P T E N-3^{\prime} \mathrm{UTR}$, was replaced by GAATTC, the EcoRI site. In the second plasmid, WT-miR-494-GLO-MT2-PTEN-3'UTR, the seed region of the second predicted binding site at $P T E N-3^{\prime} \mathrm{UTR}$, was replaced by GAATTC, the EcoRI site. Luciferase assays were done in NK92 cells transfected with plasmids with WT, MT1, or MT2 PTEN-3'UTR.

A plasmid was derived from the plasmid WT-miR-142GLO-RICTOR-3'UTR to localize the miR-142-3p binding on the RICTOR-3'UTR (Supplemental Figure S1 and Table 1). In the plasmid, WT-miR-142-GLO-MT-RICTOR$3^{\prime} \mathrm{UTR}$, the predicted binding site at RICTOR-3'UTR, was replaced by CCGCGG, the SacII site. Luciferase assays were done in YT cells transfected with plasmids with WT or MT RICTOR-3'UTR.

\section{Plasmid for Antagomirs to miR-494-3p or miR-142-3p}

Antagomir to miR-494-3p or miR-142-3p was inserted into the miRNA cloning site in the miRCS-GLO-targetCS vector (Supplemental Figure S1 and Table 1). The target cloning site was left empty.

The vector expressing antagomir to miR-494-3p was transfected into YT cells, and Western blot analysis for PTEN was performed. The vector expressing antagomir to miR-142-3p was transfected into NK92 cells, and Western blot analysis for RICTOR was performed.

\section{Western Blot Analysis}

Western blot analysis was performed with antibodies against luciferase (rabbit polyclonal) from GeneTex (San Antonio, TX); TP53 (rabbit polyclonal) from Abcam
(Cambridge, MA); PTEN (number 9552, rabbit polyclonal), RICTOR (number 2140, rabbit polyclonal), and pAKT (Ser473) (D9E, rabbit monoclonal) from Cell Signaling (Danvers, MA); and T-bet (4B10, mouse monoclonal) and glyceraldehyde-3-phosphate dehydrogenase (FL-355, rabbit polyclonal) from Santa Cruz Biotechnology (Santa Cruz, CA).

\section{Chromatin IP for Binding of T-bet on the PTEN Promoter or Binding of TP53 on the miR-142-3p Promoter}

Chromatin immunoprecipitation (IP) was done with the SimpleChIP assay on YT or YT-T-bet cells. Briefly, YT or YT-T-bet cells were fixed in $1 \%$ formaldehyde for crosslinking. After lysis of the cells, the nuclei were pelleted, and the chromatins were digested with micrococcal nuclease.

For binding of T-bet to the PTEN promoter, the chromatins were reacted with a T-bet antibody (mouse monoclonal, clone 4B10; Santa Cruz Biotechnology) or mouse IgG as negative control, or a mouse antibody against histone 3 (MABI 0301; Active Motif, Carlsbad, CA) as a positive control. Protein G magnetic beads were used for isolation of the chromatin, and protein-DNA cross-linking was reversed with proteinase $\mathrm{K}$. The DNAs were amplified with the following primers: $5^{\prime}$-AGCTGAGGTCCCAGACATGTGGTA-3' (forward) and 5'-AGCTGGACAGGTCGGCTCCCCAAT-3' (reverse).

For binding of TP53 on the mir-142-3p promoter, the chromatin was reacted with a TP53 antibody (rabbit polyclonal; Abcam) or rabbit IgG as negative control, or a rabbit antibody against histone 3 (D2B12, rabbit monoclonal; Cell Signaling) as positive control. The DNAs were purified and amplified with the following primers: 5'-GGGGATGGGGTGGAGCCTTTA-3' (forward) and 5'-CTCCTTGTGGCTTCCTAAGAT-3' (reverse).

Quantitative RT-PCR for miR-494-3p, Anti-miR-494-3p, miR-142-3p, and Anti-miR-142-3p

Total RNAs were extracted from cells with the TRIzol method (Sigma-Aldrich, St. Louis, MO). A stem-loop RTPCR was performed according to a published protocol. ${ }^{1,2}$ For RT, a specific reverse primer, $5^{\prime}$-N44-GAGGTT-3' for miR-494-3p, 5'-N44-TGAAAC-3' for anti-miR-494-3p, $5^{\prime}$-N44-TCCATA-3' for miR-142-3p, or 5'-N44-TGTAGT-3' for anti-miR-142-3p, was used, where N44 is the stem-loop 5'-GTCGTATCCATGGCAGGGTCCGAGGTATTCGCCATGGATACGAC- $3^{\prime}$. For PCR, a specific forward PCR primer, 5'-GGGTGAAACATACACGGGAA-3' for miR-4943p, 5'-AAAGAGGTTTCCCGTGTATG-3' for anti-miR-4943p, 5'-GGGTGTAGTGTTTCCTACTT-3' for miR-142-3p, or $5^{\prime}$-GGGTCCATAAAGTAGGA-3' for anti-miR-142-3p, and a universal reverse PCR primer, $5^{\prime}$-TGGCAGGGTCCGAGGT- $3^{\prime}$, were used. The difference between the threshold cycles of miR-494-3p, anti-miR-494-3p, miR-142-3p, or anti-miR$142-3 p$, and U6 snRNA [dCT $\left(\mathrm{CT}_{\text {miRNA }}-\mathrm{CT}_{\mathrm{U} 6}\right)$ ] was 
Table 1 Cloning Sites for miRNAs and 3'UTRs

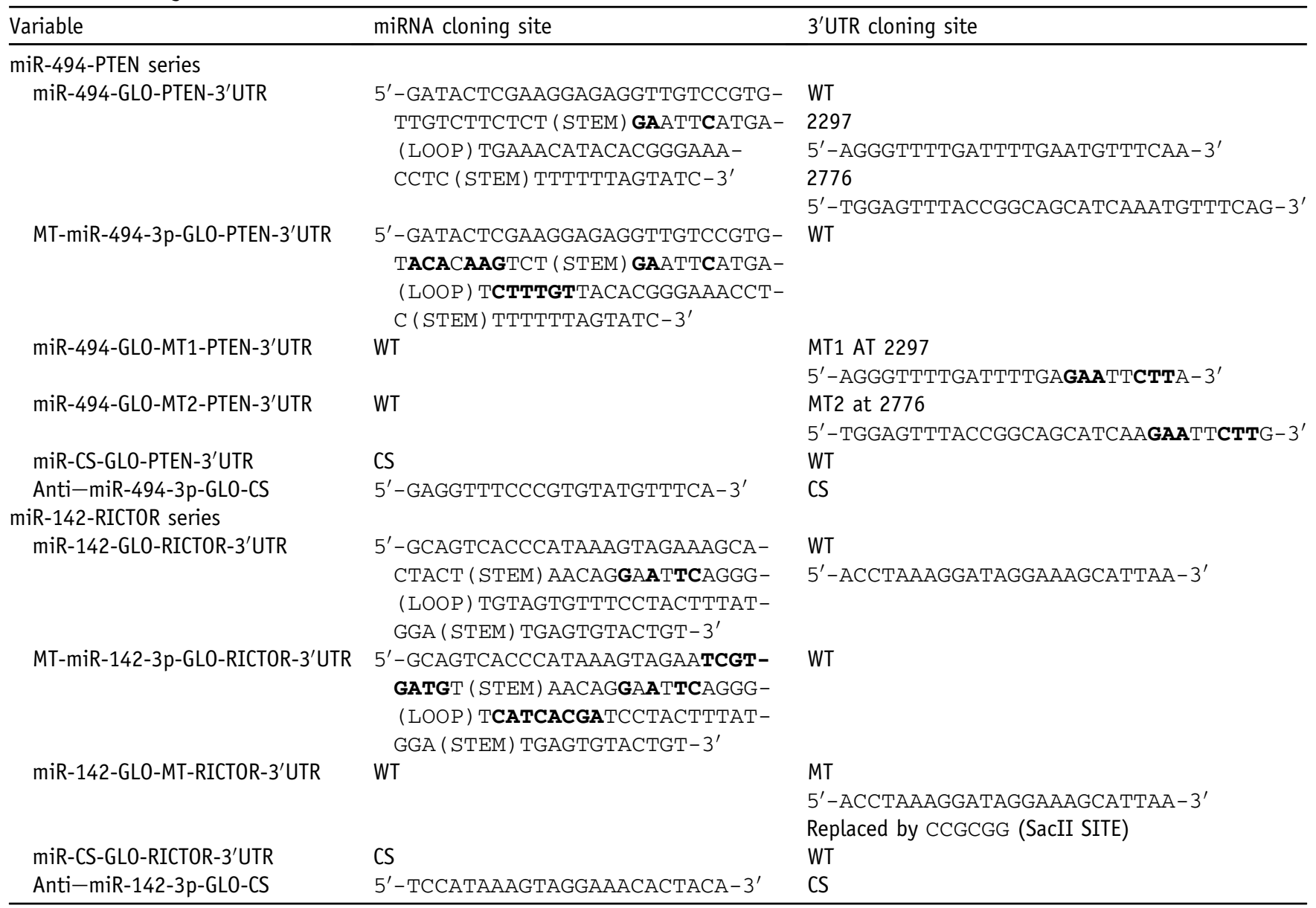

miRNA cloning site: $5^{\prime}$-PacI-TTTTTTGAATTCTTTTTT-AscI-3' $3^{\prime} 3^{\prime}$ UTR cloning site: $5^{\prime}$-NheI-AAAAAAAAAAAAAAAAA-SalI- $3^{\prime}$. Mutations are boldfaced. Mutations in the loop are artificial restriction sites for cloning. Mutations outside the loop are for testing bindings between miRNAs and mRNAs.

CS, cloning site; GLO, firefly luciferase coding region; MT, mutant; PTEN, phosphatase and tensin homolog; RICTOR, RPTOR-independent companion of mammalian target of rapamycin complex 2; UTR, untranslated region; WT, wild type.

calculated from the mean values of duplicate measurements. This definition of dCT implies an inverse correlation between dCTs and miRNA levels.

Caspase Activities in YT and NK92 Cell Lines Treated with AKT Activator SC79 or AKT Inhibitor GSK690693

NK92, NK92 transfected with miR-494-3p, or NK92 transfected with anti-miR-142-3p was treated with an AKT activator SC79 (Sigma-Aldrich, St. Louis, MO). YT, YT transfected with miR-142-3p, or YT transfected with anti-miR-494-3p was treated with an AKT inhibitor GSK690693 (Sigma-Aldrich). Caspase activities were measured with a luminescent assay Caspase-Glo 3/7 (Promega, St. Louis, MO), in which the luciferase substrate aminoluciferin is generated from Z-DEVD-aminoluciferin after caspase cleavage.

Briefly, 10,000 cells were grown in $200 \mu \mathrm{L}$ medium containing $25 \mu \mathrm{mol} / \mathrm{L} \mathrm{SC79}$ or GSK690693 for 24 hours.
An equal volume of reagent containing reagents for lysis, the substrate Z-DEVD-aminoluciferin, and luciferase were added. The mixture was incubated at $25^{\circ} \mathrm{C}$ for 30 minutes, and the luminescence was measured.

\section{Immunohistochemistry for PTEN, RICTOR, and pAKT}

Immunoperoxidase stains on formalin-fixed, paraffinembedded tissue sections with antibodies against PTEN (number 31392, rabbit polyclonal; Abcam), RICTOR (number 56578, mouse monoclonal; Abcam), and pAKT (Ser473) (D9E, rabbit monoclonal; Cell Signaling). Antigen retrieval was performed in Tris buffer at $\mathrm{pH}$ 8. The primary antibody was applied to the slides at $37^{\circ} \mathrm{C}$ for 45 minutes. Biotinconjugated secondary antibodies, peroxidase-conjugated streptavidin, and 3,3' diaminobenzidene were used sequentially for completing the reactions. The percentages of positive cells, as averages of 10 randomly selected high-power fields, were determined. 


\section{Results}

NNLs Express Only a Limited Set of miRNAs

The NanoString technology (Seattle, WA) was used to determine the expression profiles of 651 miRNAs in a series of 36 NNLs. The top 20 miRNAs account for approximately $50 \%$ of the miRNA transcripts (Figure 1A and Supplemental Table S1). Because human NK cells express approximately 50 miRNAs and the top 10 miRNAs of murine NK cells constitute $65 \%$ of all miRNA transcripts, ${ }^{3,4}$ malignant NNLs are similar to normal NK cells in that they all express only a limited set of miRNAs.

\section{miRNAs in NNLs Regulate the AKT Pathway}

Pathway analysis for the top five miRNAs in NNLs, miR-21, miR-142-3p, miR-126, miR-451, and miR-494-3p, was performed on the DIANA-mirExTra web server (http://diana. cslab.ece.ntua.gr/hexamers, last accessed January 23, 2015). ${ }^{24}$ Except for miR-142-3p, four of the five top miRNAs have been shown experimentally to regulate the AKT pathway (Figure 1B and Table 2). ${ }^{26-33}$ In addition, a target site for miR$142-3 p$ on the $3^{\prime}$ UTR of RICTOR was identified with the RNAhyb program. ${ }^{25}$

These miRNAs may either activate or inactivate the AKT pathway (Table 2). For example, both miR-126-3p and miR142-3p inhibit the AKT pathway, whereas miR-494-3p, miR-21-5p, and miR-451-5p all activate the AKT pathway. We are interested in the inhibition of PTEN by miR-494-3p, because of the importance of the AKT pathway in nasal lymphoma, ${ }^{6-8}$ and in miR-142-3p, because the regulation of RICTOR by miR-142-3p is a novel finding.

\section{miR-494-3p/PTEN/pAKT Pathway}

Overexpression of miR-494-3p Inhibits PTEN with Secondary Up-Regulation of pAKT in NK92 Cells

Two binding sites for miR-494-3p at the $3^{\prime}$ UTR of PTEN mRNA were identified with the RNAhyb online program (Figure 2A). ${ }^{33}$ The $5^{\prime}$ end of the first binding site is located at the $2297^{\text {th }}$ nucleotide after the stop codon. This is a strong model, because of the perfect matches at the seed region (ie, between the second and eighth nucleotides at the $5^{\prime}$ end of miR-494-3p and the $3^{\prime}$ end of the target, and also because of the presence of only small internal mismatches of at most dinucleotides but not large internal bulges). The second binding site is located at the $2776^{\text {th }}$ nucleotide after the stop codon. This is a weak model, because of significant mismatches at the $5^{\prime}$ end and the internal bulges.

In NK92 cells, overexpression of miR-494-3p, but not mutant miR-494-3p, inhibited the expression of luciferase with wild-type PTEN-3'UTR. In addition, miR-494-3p inhibited $P T E N-3^{\prime}$ UTR with a mutated second binding site but not $P T E N-3^{\prime}$ UTR with a mutated first binding site (Figure 2B). These data confirmed the first binding site for miR-494-3p. In
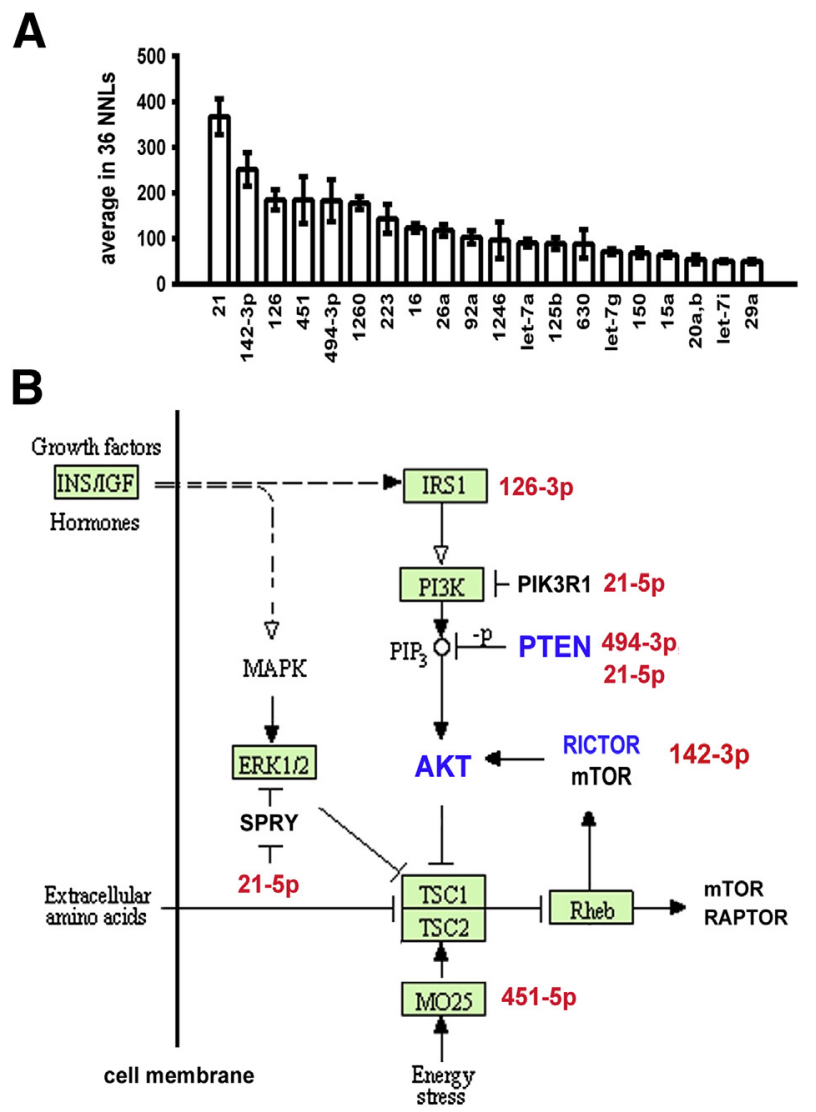

Figure 1 Identification of a PTEN/AKT/mTOR-RICTOR pathway. A: Distribution of the top 20 miRNAs in 36 nasal NK cell lymphomas (NNLs) shows that NNLs express only a limited set of miRNAs. The top 20 miRNAs account for approximately $50 \%$ of the reads. Of the top 20 miRNAs in NNLs, only 10 miRNAs have an average level $>100$ in the 36 cases. For $y$ axis, relative expression level; $x$ axis, miRNAs. B: miRNAs in NNLs regulate the AKT pathway. The top five miRNAs in NNLs are miR-21, miR-142-3p, miR-126, miR-451, and miR-494-3p. On the DIANA-mirExTra web server (http://diana.cslab.ece.ntua.gr/hexamers, last accessed January 23, 2015), ${ }^{24}$ four of the five top miRNAs, except miR-142$3 p$, regulate the AKT pathway. However, the RNAhyb program ${ }^{25}$ predicted RICTOR as a target of miR-142-3p. Arrows indicates stimulation; bars, inhibition; solid lines, direct interaction; dashed lines, indirect interactions. miRNAs are red; targets, blue or black. ERK, extracellular signal-regulated kinase; IGF, insulin growth factor; INS, insulin; MAPK, mitogen-activated protein kinase; PIP3, phosphatidylinositol 3,4,5 triphosphates; PI3K, phosphatidylinositol 3-kinase; SPRY, sprouty homolog; TSC, tuberous sclerosis.

addition, Western blot analysis showed miR-494-3p, but not a mutant, inhibited expression of luciferase-PTEN-3'UTR in NK92 cells (Figure 2C).

For further confirmation, a plasmid expressing miR494-3p was transfected into NK92 cells, and a plasmid expressing anti-miR-494-3p was transfected into YT cells. NK92 cells and YT cells are lymphoma cells of NK origin. NK92 cells have higher expression of PTEN and lower expression of pAKT than YT cells. As predicted, miR-494$3 \mathrm{p}$ inhibited PTEN with secondary induction of pAKT in NK92 cells, whereas anti-miR-494-3p induced PTEN with secondary inhibition of pAKT in YT cells (Figure 2D).

Functionally, pAKT ${ }^{\text {weak }}$ NK92 cells are more sensitive to the AKT activator SC79 than pAKT ${ }^{\text {strong }}$ NK92-miR-494-3p cells. In contrast, pAKT $^{\text {strong }}$ YT cells are more sensitive to the 
Table 2 Regulation of the AKT Pathway by miRNAs

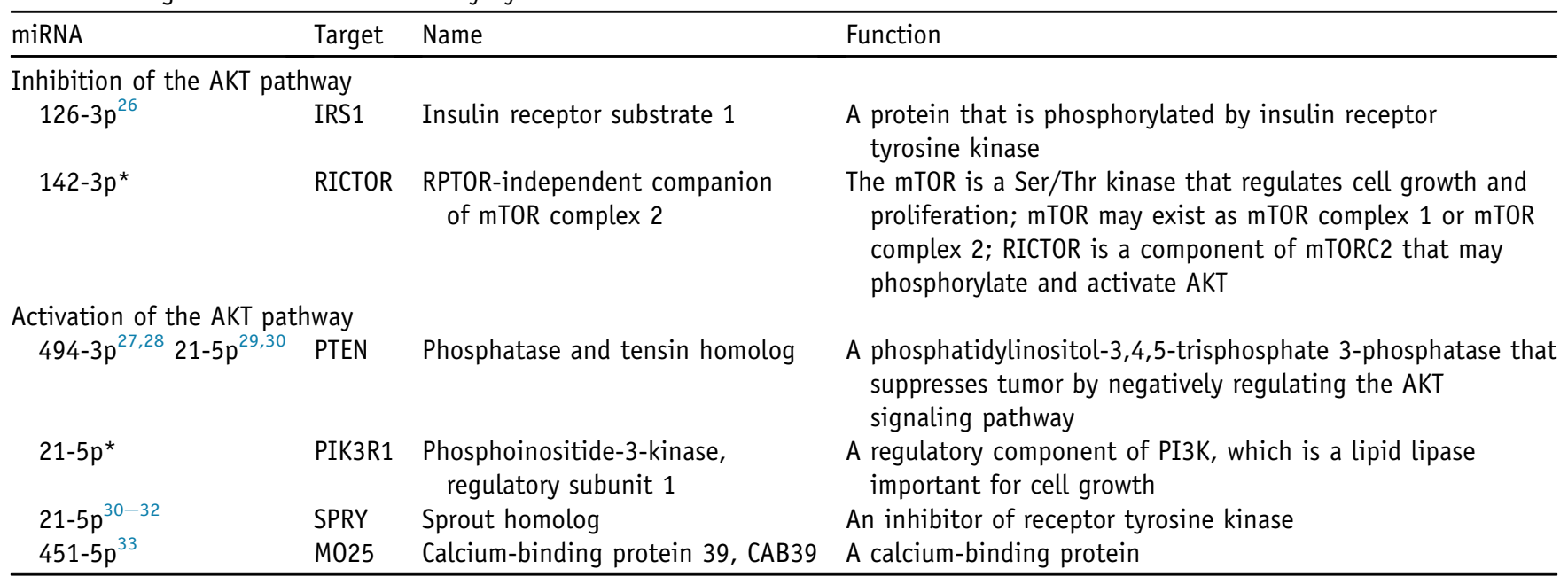

${ }^{*}$ A predicted target by RNAhyb or http://www. microrna.org.

AKT inhibitor GSK690693 than pAKT ${ }^{\text {weak }}$ YT-anti-miR494-3p cells (Figure 2E).

Overexpression of miR-142-3p Inhibits RICTOR with Secondary Down-Regulation of pAKT in YT Cells A binding site for miR-142-3p at the $3^{\prime} \mathrm{UTR}$ of RICTOR mRNA was identified with the RNAhyb online program (Figure $3 \mathrm{~A}$ ). ${ }^{25}$ The $5^{\prime}$ end of the binding site is located at the $864^{\text {th }}$ nucleotide after the stop codon. Luciferase assay showed that miR-142-3p, but not a mutant, inhibited expression of luciferase-RICTOR-3'UTR in YT cells. In addition, miR-142-3p inhibited luciferase-RICTOR-3'UTR, but not luciferase-MT-RICTOR-3'UTR (Figure 3B).

For further confirmation, a plasmid expressing miR-142-3p was transfected into YT cells, and a plasmid expressing antimiR-142-3p was transfected into NK92 cells. YT cells have higher pAKT than NK92 cells, although the levels of RICTOR are approximately equal. As predicted, miR-142-3p inhibited RICTOR with secondary suppression of pAKT in YT cells, whereas anti-miR-142-3p induced RICTOR with secondary induction of AKT in NK92 cells (Figure 3C).

In the AKT-mTOR pathway, mTOR not only mediates the oncogenic effects of pAKT, but RICTOR in the mTOR complex 2 may phosphorylate and activate AKT. ${ }^{16}$ Therefore, there is possibly a miR-142-3p-RICTOR-AKT pathway in NNL.

Functionally, $\mathrm{pAKT}^{\text {strong }}$ YT cells are more sensitive to the AKT inhibitor GSK690693 than pAKT ${ }^{\text {weak }}$ YT-miR142-3p cells. In contrast, pAKT $^{\text {weak }}$ NK92 cells are more sensitive to the AKT activator SC79 than $\mathrm{pAKT}^{\text {strong }}$ NK92-anti-miR-142-3p cells (Figure 3D).

\section{T-bet Induces PTEN and TP53 to Inhibit AKT in YT-T-bet Cells}

We showed previously that the EBV-encoded miRBART20-5p inhibits Tbet with secondary suppression of
TP53 in NNL. ${ }^{1,2}$ Because a genome-wide screen identified the promoter of PTEN as one of the T-bet binding sites, ${ }^{34,35}$ there might be interactions between the miR-BART20-5pT-bet-TP53 pathway and the miR-494-3p-PTEN-AKT pathway.

A model for the interactions between the transcription factor T-bet and the promoter of PTEN is shown in Figure 4A. The $5^{\prime}$ end of the binding site is at position -6101 before the transcription start site. YT cells are lymphoma cells of NK cell origin. These cells express no or weak T-bet. Chromatin IP was done in YT cells and YT cells transfected with a plasmid expressing T-bet (YT-T-bet cells). Consistent with the model, there was stronger binding of T-bet to the PTEN promoter in YT-T-bet cells than in YT cells (dCT: 7.8 in YT-T-bet versus 13.6 in YT) (Figure 4B).

Furthermore, Western blot analysis showed that T-bet induces PTEN and TP53 with inhibition of pAKT in YT-T-bet cells (Figure 4C). Because inhibition of AKT by PTEN and TP53 is well established, ${ }^{12,13,15}$ we conclude that T-bet induces both PTEN and TP53 to inhibit AKT in YT-T-bet cells.

\section{Tumor Suppressor TP53 Induces miR-142-3p}

Figure $4, \mathrm{~A}-\mathrm{C}$, demonstrates a connection between the T-bet-TP53 pathway and the miR-494-3p-PTEN-AKT pathway. Because of the down-regulation of RICTOR in YT-T-bet cells (Figure 4C), TP53 might directly regulate miR-142-3p ${ }^{36}$ and secondarily the RICTOR-pAKT pathway.

The expression level of miR-142-3p was higher in YT-T-bet cells than in YT cells (dCT: 0.45 in YT-T-bet versus 2.72 in YT) (Figure 4D). This finding supported indirect induction of miR-142-3p by T-bet through TP53.

A binding site for TP53 on the promoter of miR-142-3p was noted. ${ }^{36}$ The $5^{\prime}$ end of the binding site was at position -166 before the transcription start site (Figure 4E). The 
A position 2297

target 5' A

GGGUUUU UU UG AUGUUUCA

UCCAAAG GG AC UACAAAGU

$$
\begin{array}{ll}
3^{\prime} \mathrm{C} & \text { C } A \\
& \text { miR-494-3p }
\end{array}
$$

B

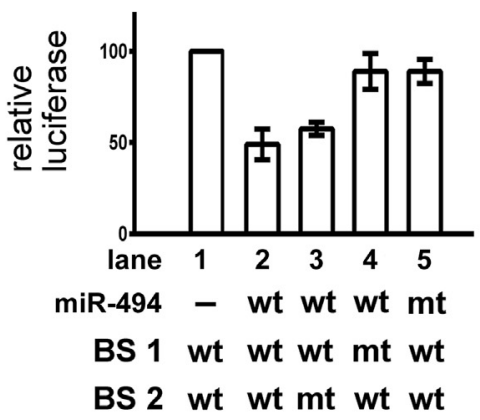

BS2

position 2776

target 5' U A UA GCA CA CAA G 3'

$\begin{array}{llllll}\text { GG GUU } & \text { CCG } & \text { G } & \text { U } & \text { AUGUUUCA } \\ \text { UC CAA } & \text { GGC } & \text { C } & \text { A } & \text { UACAAAGU } \\ \text { C } & \text { AG } & \text { A } & & & \\ & & & & & \text { mir-494-3p }\end{array}$

C

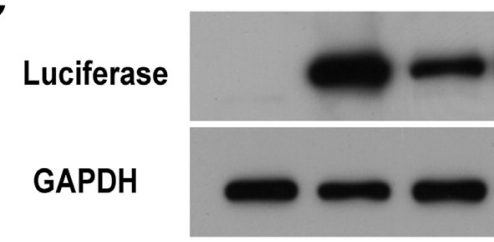

$\begin{array}{llll}\begin{array}{l}\text { Luciferase- } \\ \text { PTEN 3'UTR }\end{array} & - & \text { wt } & \text { wt } \\ \text { miR-494-3p } & - & \text { mt } & \text { wt }\end{array}$

E
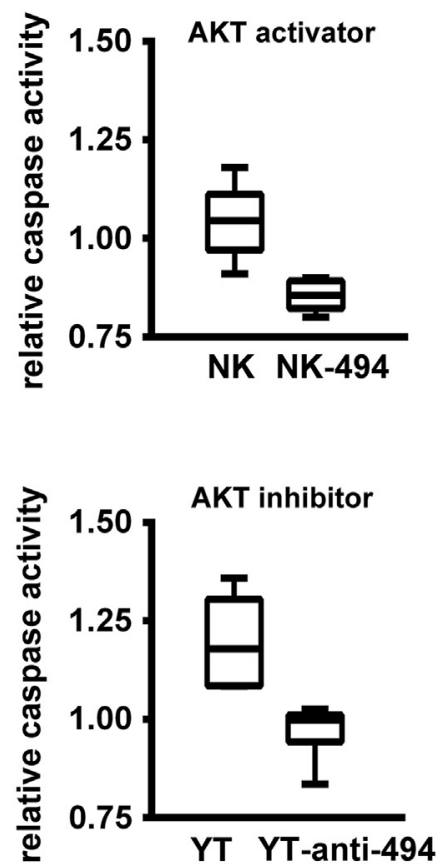

Figure 2 A miR-494-3p/PTEN/pAKT pathway: Overexpression of miR-494-3p inhibits PTEN with secondary up-regulation of pAKT in NK92 cells. A: Models for the interactions between miR-494-3p and the $3^{\prime}$ untranslated region (UTR) of PTEN mRNA. The $5^{\prime}$ end of the target site is predicted to be located at the $2297^{\text {th }}$ or $2776^{\text {th }}$ nucleotide after the stop codon. B: Confirmation of the first model with a luciferase assay in NK92 cells. The activity of luciferase is inhibited in NK92 cells with transfections of both a wild-type (WT) miR-494-3p and a wild-type first binding site at position 2297 (lanes 1 and 2). The inhibition is not removed by a mutated second binding site at position 2776 (lane 3), but is removed by mutations at either the first binding at position 2297 or miR-494-3p (lanes 4 and 5). C: Confirmation of the model with Western blot analysis for luciferase in NK92 cells. Wild-type miR-494-3p, but not mutant (MT) miR-494-3p, inhibits the expression of luciferase-PTEN-3'UTR in NK92 cells. D: 0verexpression of miR-494-3p inhibits PTEN with secondary induction of pAKT in NK92 cells, whereas overexpression of anti-miR-494-3p induces PTEN with secondary suppression of pAKT in YT cells. Western blot analysis in NK92 overexpressing miR494-3p or YT cells overexpressing anti-miR-494-3p supports miR-494-3p/PTEN/pAKT pathway, in which miR-494-3p inhibits PTEN and PTEN inhibits pAKT (left column). Quantitative RT-PCR for miR-494-3p or anti-miR-494-3p. For $x$ axis, cell lines; $y$ axis, dCT for miR-494-3p (5.8 in NK versus 0.89 in NK-miR-494$3 p$ ) and dCT for anti-miR-494-3p (17.6 in YT versus 9.7 in YT-anti-miR-494-3p) (right column). E: NK92 cells are more sensitive to the AKT activator SC79 than NK92-miR-494-3p cells (median caspase activity, 1.04 versus $0.85 ; P=0.005$ ). In contrast, YT cells are more sensitive to the AKT inhibitor GSK690693 than YT-anti-miR-494-3p cells (median caspase activity, 1.18 versus $1.00 ; P=0.005$ ). For $y$ axis, caspase activity in cells treated with the drug relative to untreated cells. GAPDH, glyceraldehyde-3-phosphate dehydrogenase. 
A

RICTOR-3'UTR

position 864

$\begin{array}{lllll}\text { A } & \text { GA } & \text { A } & \text { A } & 3^{\prime}\end{array}$

CC UAAAG UAGG AAGCAUUA

GG AUUUC AUCC UUUGUGAU

$3^{\prime} \mathrm{A} \mathrm{U}$

GU $5^{\prime}$
B



LuciferaseRICTOR-3'UTR

miR-142-3p

- wt mt wt
C

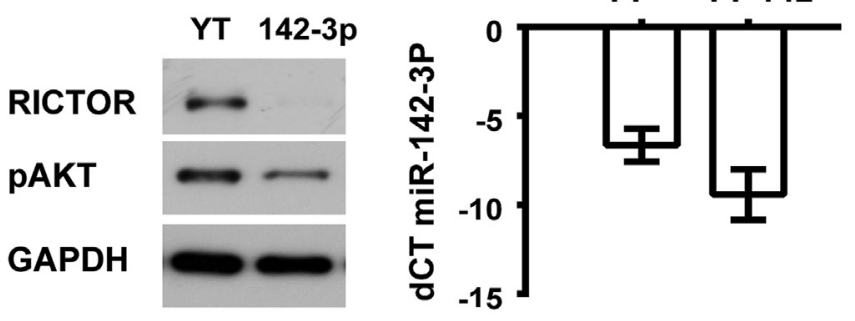

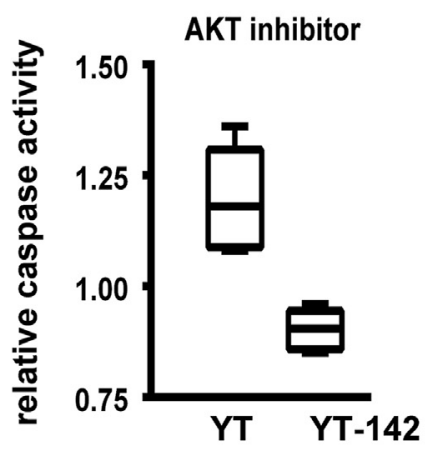

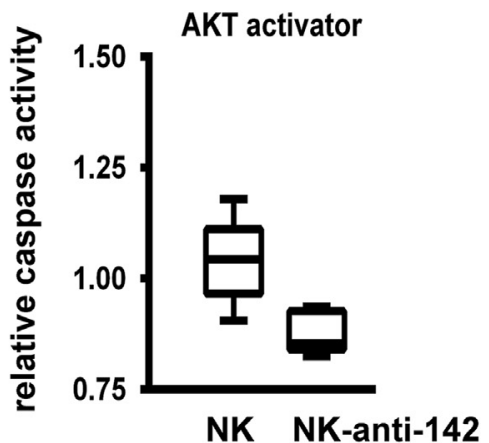

Figure 3 miR-142-3p/RPTOR-independent companion of mammalian target of rapamycin complex 2 (RICTOR)/phosphorylated AKT (pAKT) pathway: overexpression of miR-142-3p inhibits RICTOR with secondary down-regulation of pAKT in YT cells. A: Models for the interactions between miR-142-3p and the $3^{\prime}$ untranslated region (UTR) of RICTOR mRNA. The $5^{\prime}$ end of the target site is predicted to be located at the $864^{\text {th }}$ nucleotide after the stop codon. B: Confirmation of the model with a luciferase assay in YT cells. In YT cells, the activity of luciferase was inhibited only with transfections of both wild-type (WT) miR-142-3p and a wild-type binding site at position 864. The inhibition was removed when either miR-142-3p or the binding site was mutated. C: 0verexpression of miR-142-3p inhibits RICTOR with secondary suppression of pAKT in YT cells, whereas overexpression of anti-miR-142-3p induces RICTOR with secondary induction of pAKT in NK92 cells. Western blot analysis in YT cells overexpressing miR-142-3p or in NK92 cells overexpressing anti-miR-142-3p supports a miR-142-3p/RICTOR/pAKT pathway, in which miR-142-3p inhibits RICTOR and RICTOR induces pAKT (left panel). Quantitative RT-PCR for miR-142$3 p$ or anti-miR-142-3p. For $x$ axis, cell lines; $y$ axis, dCT for miR-142-3p (-6.6 in YT versus -8.7 in YT-miR-142-3p) and dCT for anti-miR-142-3p (16.9 in NK92 versus 2.2 in NK92-anti-miR-142-3p) (right panel). D: YT cells are more sensitive to the AKT inhibitor GSK690693 than YT-miR-142-3p cells (median caspase activity, 1.18 versus $0.91 ; P=0.005$ ). In contrast, NK92 cells are more sensitive to the AKT activator SC79 than NK92-anti-miR-142-3p cells (median caspase activity, 1.04 versus. $0.85 ; P=0.01$ ). For $y$ axis, caspase activity in cells treated with the drug relative to untreated cells. GAPDH, glyceraldehyde-3phosphate dehydrogenase; MT, mutant; NK, natural killer.

model was confirmed with chromatin IP that showed stronger binding of TP53 to the miR-142-3p promoter in YT-T-bet cells than in YT cells (dCT: 7.9 in YT-T-bet versus 12.7 in YT) (Figure 4F).
These data provide additional evidence that TP53 may regulate miR-142-3p. However, this finding does not exclude additional regulators on the promoter of miR-142-3p. 
A

T-bet binding site

on the PTEN promoter

.6101 5'-AAATTCACAGC-3'

CONSENSUS $\quad 5^{\prime}$-AATITICACACC-3'
B

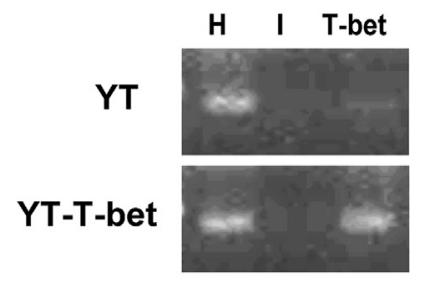

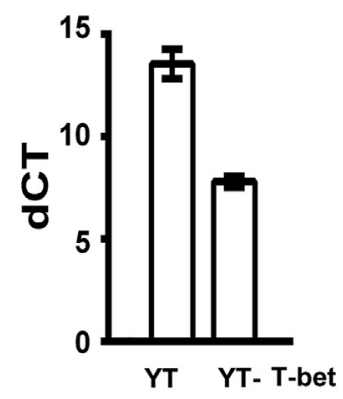

D

C

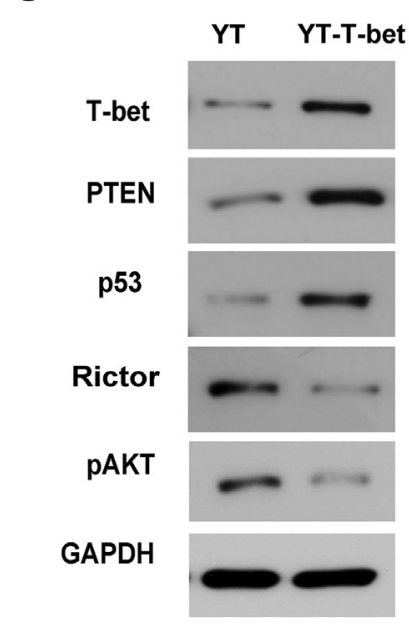

E







$\mathbf{F}$



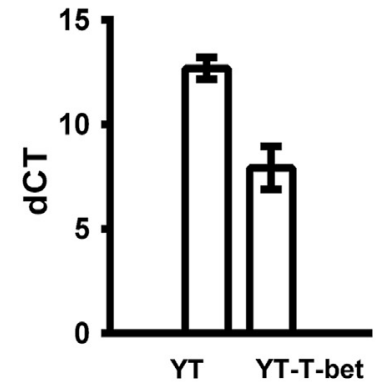

Figure 4 Overexpression of T-bet in YT cells inhibits AKT indirectly through pentaerythritol tetranitrate (PTEN), TP53, and RPTOR-independent companion of mammalian target of rapamycin complex 2 (RICTOR). A: The transcription factor T-bet controls the PTEN promoter. The $5^{\prime}$ end of the binding site is at position - 6101 before the transcription start site. Comparison between the binding site and the consensus T-box binding site. B: Confirmation of the model with chromatin immunoprecipitation (IP). Chromatin IP was performed in YT cells and YT-T-bet cells, with antibodies against histone (H) as a positive control, T-bet, or an isotype (I) as a negative control. Note the stronger binding of T-bet to the PTEN promoter in YT-T-bet cells than in YT cells (dCT: 13.6 in YT versus 7.80 in YT-T-bet). Quantitative PCR on DNA precipitated by an antibody against either histone or T-bet was performed with the same primers targeting the T-bet binding site. The dCT was calculated. C: T-bet induces PTEN and TP53 with inhibition of RICTOR and pAKT in YT-T-bet cells. Western blot analysis confirmed induction of PTEN and TP53 with secondary inhibition of RICTOR and PAKT by T-bet. D: Indirect induction of miR-142-3p by T-bet. YT-T-bet cells have higher levels of miR-142-3p than YT cells (dCT: 2.72 in YT versus 0.45 in YT-T-bet). E: The transcription factor TP53 controls the miR-142-3p promoter. The $5^{\prime}$ end of the binding site is at position -166 before the transcription start site. The consensus TP53 binding site is shown below. F: Confirmation of the model with chromatin IP. Chromatin IP was performed in YT cells and YT-T-bet cells, with antibodies against $\mathrm{H}$ as a positive control, TP53, or an I as a negative control. Note the stronger binding of TP53 to the miR-142-3p promoter in YT-T-bet cells than in YT cells (dCT: 12.7 in YT verssu 7.9 in YT-T-bet). Quantitative PCR on DNA precipitated by an antibody against either histone or TP53 was performed with the same primers targeting the TP53 binding site. The dCT was calculated. GAPDH, glyceraldehyde-3-phosphate dehydrogenase.

miR-494 Correlates Inversely with PTEN, and miR-142-3p Correlates Inversely with RICTOR in NNLs

Immunohistochemical stains for PTEN, RICTOR, and pAKT were performed on 24 NNLs. Representative immunostains are shown in Figure 5, A-F.
The levels of miRNAs were measured with the NanoString technology. There is an inverse correlation between miR-494-3p and miR-142-4p in 36 NNLs $(r=-0.47$, $P=0.003)$. NNLs can be classified into $18 \mathrm{miR}-494^{\text {low }} /$ miR-142 ${ }^{\text {high }}$ NNLs and $18 \mathrm{miR}-494^{\text {high }} / \mathrm{miR}-142^{\text {low }}$ NNLs (Figure 5A). 


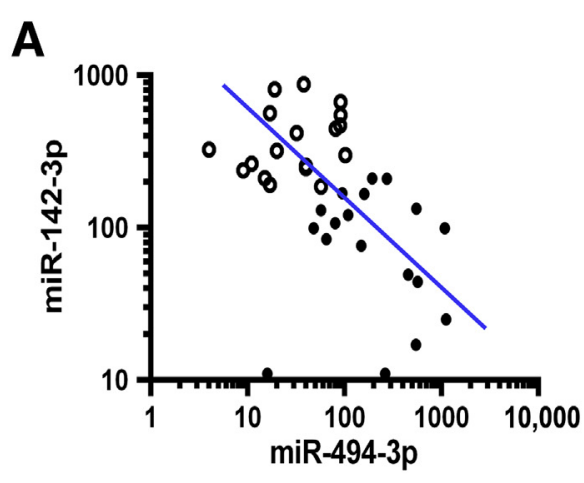

H

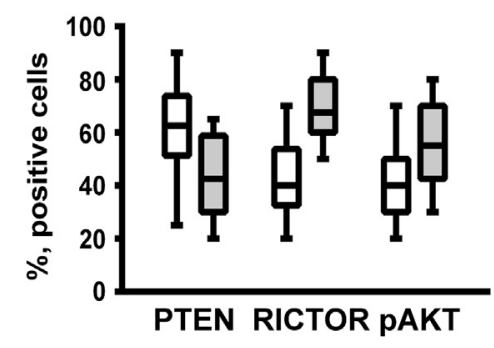

I
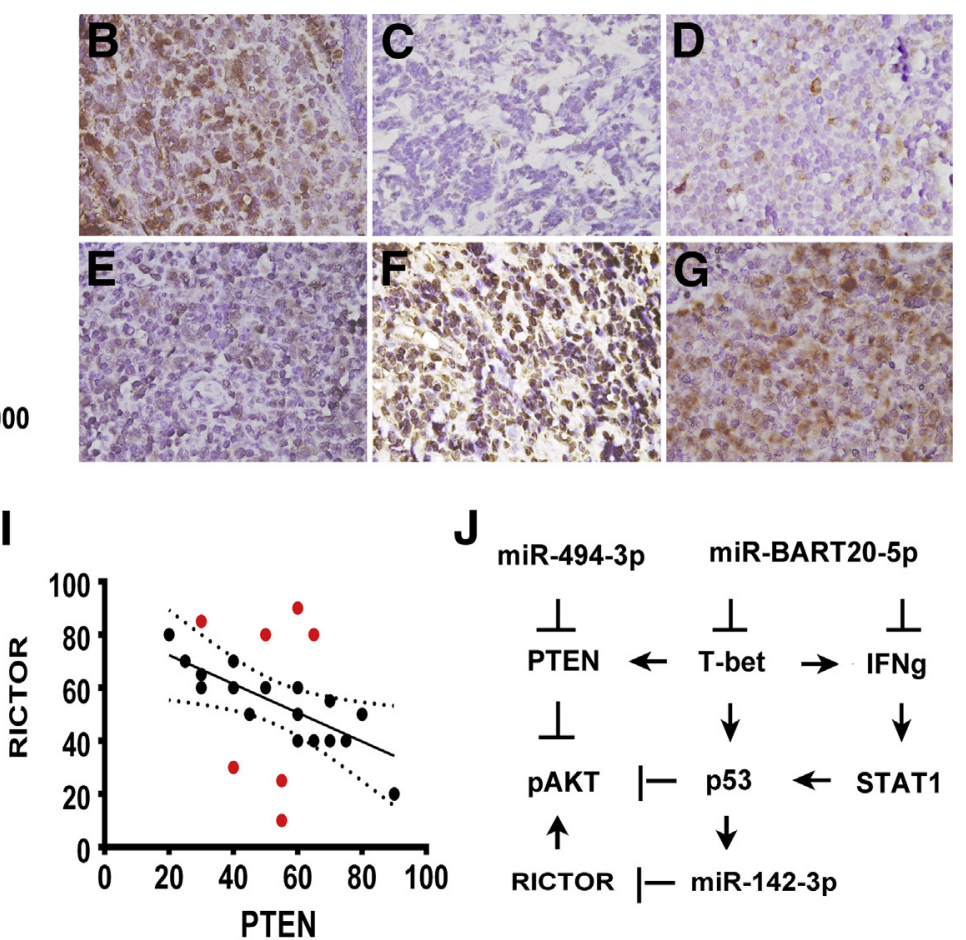

$\mathrm{J}$ miR-494-3p miR-BART20-5p

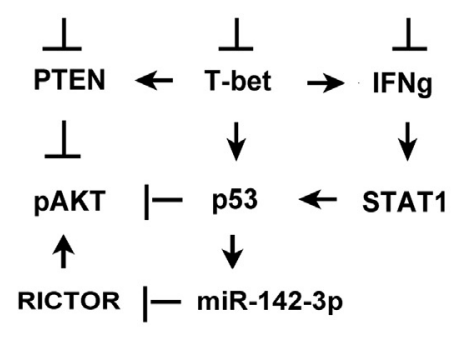

Figure 5 miR-494 correlates inversely with pentaerythritol tetranitrate (PTEN), and miR-142-3p correlates inversely with RPTOR-independent companion of mammalian target of rapamycin complex 2 (RICTOR) in nasal natural killer (NK) cell lymphomas (NNLs). A: An inverse correlation between miR-494-3p and miR-12-3p in NNLs (Pearson correlation coefficient $r=-0.47, P=0.003$ ). For $x$ axis, levels of miR-494-3p; $y$ axis, levels of miR-142-3p (white circle, $\mathrm{miR}-44^{\text {low }} / \mathrm{miR}^{-142^{\text {high }}}$ NNLs; black circle, miR-494 ${ }^{\text {high }} / \mathrm{miR}-142^{\text {low }}$ ). B-G: Representative immunostains for PTEN, RICTOR, and phosphorylated AKT (pAKT) in NNLs. B-D: A PTEN ${ }^{+}$RICTOR $^{-} /$pAKT $^{-}$NNL. E-G: A PTEN ${ }^{-} /$RICTOR $^{+} /$pAKT $^{+}$NNL. An Olympus BX60 microscope with a $10 \times$ ocular lens and a $100 \times$ objective lens was used (Olympus, Tokyo, Japan). H: $\mathrm{miR}-494^{\text {low }} / \mathrm{miR}-142^{\text {high }}$ NNLs (white) have stronger PTEN but weaker RICTOR and pAKT than miR-494 ${ }^{\text {high }} / \mathrm{miR}$ $142^{\text {low }}$ NNLs (gray). Box-and-whisker plots for the percentages of positive cells for PTEN, RICTOR, and pAKT. I: PTEN correlates inversely with RICTOR. The expression of PTEN correlates inversely with the expression of RICTOR ( $r=-0.46, P=0.02$ for all 24 cases). For $x$ axis, percentages of PTEN ${ }^{+}$cells; $y$ axis, percentages of RICTOR ${ }^{+}$cells. Red circles, seven cases outside the $95 \%$ CI; black circles, 17 cases inside the $95 \%$ CI. J: A regulatory circuit in NNL. Arrowhead indicates increase; bar, decrease. In this study, we showed that miR-494-3p inhibits PTEN with secondary increase of pAKT in NNL, and miR-142-3p inhibits RICTOR with secondary suppression of pAKT in NNL. In previous studies, we showed that miR-BART20-5p inhibits T-bet and IFN- $\gamma$ with secondary suppression of TP53. ${ }^{1,2}$ Note the T-bet-TP53 pathway may inhibit AKT through PTEN and miR-142-3p.

Immunohistochemistry for PTEN, RICTOR, and pAKT was performed on $12 \mathrm{miR}-494^{\mathrm{low}} / \mathrm{miR}-142^{\text {high }} \mathrm{NNLs}$ and 12 miR-494 $4^{\text {high }} / \mathrm{miR}-142^{\text {low }}$ NNLs (Figure 5, B-G). The 12 miR-494 ${ }^{\text {low }} / \mathrm{miR}-142^{\text {high }}$ NNLs had higher PTEN and lower RICTOR and pAKT than the $12 \mathrm{miR}-494^{\text {high }} / \mathrm{miR}-142^{\text {low }}$ NNLs. The median percentages of positive cells were $62 \%$ versus $43 \%$ for PTEN at $P=0.01,40 \%$ versus $68 \%$ for RICTOR at $P=0.01$, and $40 \%$ versus $55 \%$ for pAKT at $P=0.03$ (Figure $5 \mathrm{H}$ ).

There is an inverse correlation between RICTOR and PTEN in the 24 cases (Figure 5I), between miR-494-3p and PTEN $(r=-0.61$ at $P=0.002)$, and between miR-142-3p and RICTOR $(r=-0.51$ at $P=0.01)$.

A Molecular Loop Regulated by miR-494-3p, miR-142-3p, and miR-BART20-5p in the Pathogenesis of NNL: T-bet Induces Both PTEN and TP53 to Inhibit AKT

To summarize, miR-494-3p-PTEN-AKT and miR-142-3pRICTOR-AKT pathways were identified in NNL. Both pathways seem to connect with the T-bet-TP53 pathway.
Previously, we showed that the EBV-encoded miR-BART20$5 \mathrm{p}$ inhibits Tbet and $I F N G$ with secondary suppression of TP53 in NNL. ${ }^{1,2}$ Taken together, cooperation among miR-BART20$5 p$, miR-494-3p, and miR-142-3p regulates a molecular loop, in which T-bet induces both PTEN and TP53 to inhibit pAKT. Modulation of the molecular loop by miRNAs is probably critical in the development of NNL (Figure 5J).

\section{Discussion}

$\mathrm{NNL}$ is an EBV-associated lymphoma that arises from the nasal mucosa. Our data show that miR-494-3p inhibits PTEN with secondary induction of AKT, whereas miR142-3p inhibits RICTOR with secondary suppression of AKT (Figure 5J). In addition, T-bet induces both PTEN and TP53, and TP53 may inhibit AKT directly ${ }^{15}$ or indirectly through the miR-142-3p/RICTOR/pAKT pathway. Significantly, the EBV-encoded miR-BART20$5 \mathrm{p}$ and human miR-494-3p cooperate to inhibit the T-bet-PTEN pathway, with secondary induction of AKT and suppression of TP53. Because TP53 reduces tumor 
growth through induction of apoptosis, this model agrees with the general conclusion that the EBV rescues tumor cells from apoptosis. ${ }^{37}$

The finding that miR-494-3p inhibits PTEN to activate AKT in NNL echoes reports that miR-494-3p inhibits PTEN in myeloid cells. ${ }^{27,28}$ Other than miR-494-3p, miR-21, ${ }^{29,30}$ one of the top 10 highly expressed miRNAs in NNLs (average level, 369 in NNLs), and miR-155, ${ }^{6}$ with an average expression level of 31 in our series, may also inhibit PTEN to activate AKT. These data support the recent conclusion on the combinatorial regulation of miRNAs: a given miRNA may have hundreds of different mRNA targets, and a given target might be regulated by multiple miRNAs. ${ }^{38}$ Because the binding for miR-21 on the $3^{\prime}$ UTR of PTEN has not been reported and is not predicted by online programs (http://www.microrna.org, last accessed January 23, 2015), and the expression of miR-155 is weak in our series, we decided to focus on the role of miR-494 in the AKT pathway.

miR-142-3p has both oncogenic and tumor-suppressing effects in various cell lines. ${ }^{39,40}$ We found that miR-142-3p may be regulated by TP53, and miR-142-3p may inhibit RICTOR with secondary suppression of the oncogene pAKT. This suggests a tumor-suppressing effect of miR142-3p; however, miR-142-3p might still be oncogenic through alternative pathways.

Our studies are based mainly on two EBV-infected lymphoma cell lines of NK cell origin, YT and NK92. These studies demonstrate the existence of a miR-494-PTEN-AKT pathway (Figure 2) and a miR-142-3p-AKT pathway (Figure 3), but do not capture the whole complexities of NNLs, as demonstrated by the finding of an inverse correlation between miR-494-3p and miR-142-3p (Figure 5H). The data from the biopsy specimens thus compensate for the inadequacy of the cell line data.

The mechanism and the biological significance of the inverse correlation are unclear. Because all NNLs are infected with EBV, miR-494-4p $\mathrm{p}^{\text {high }} / \mathrm{miR}-142-3 \mathrm{p}^{\text {low }}$ NNLs and $\mathrm{miR}-494-3 \mathrm{p}^{\text {low }} / \mathrm{miR}-142-3 \mathrm{p}^{\text {high }}$ NNLs might express different virus-encoded genes or miRNAs. Additional studies, such as genome-wide screens, will be necessary to identify these differences.

Despite these uncertainties, miR-494- $4 \mathrm{p}^{\text {high }} / \mathrm{miR}-142$ $3 \mathrm{p}^{\text {low }}$ NNLs have higher levels of pAKT than miR-494$3 \mathrm{p}^{\text {low }} / \mathrm{miR}-142-3 \mathrm{p}^{\text {high }}$ NNLs. The former group might benefit from AKT inhibitor therapy, whereas the latter group might require AKT activator therapy. ${ }^{41-43}$ Therefore, a lower AKT level does not necessarily imply a less important role when therapeutic interventions are considered.

These considerations are further supported by cell line studies. NK92 cells, which express weaker AKT than NK92-494-3p or NK92-anti-142-3p cells, are more sensitive to the AKT activator SC79. YT cells, which express stronger AKT than YT-anti-494-3p or YT-142-3p cells, are more sensitive to the AKT inhibitor GSK690693 (Figures 2E and 3D).
In addition to NNL, loss of PTEN is also present in a subset of AKT pathway-dependent diffuse large B-cell lymphomas of the germinal center type. ${ }^{44,45}$ Because miR21 inhibits PTEN, antisense miR-21 has been used to increase the cytotoxic effects of traditional doxorubicin, cyclophosphamide, vincristine, and prednisone therapy in cell lines of B-cell origin. ${ }^{46,47}$ These reports also suggest that adding antisense miR-494-3p to the standard radiochemotherapy of NNL could potentially improve the outcome of NNL. Finally, miR-494 is up-regulated by the extracellular signal-regulated kinase $1 / 2$ pathway. ${ }^{48}$ Therefore, a pharmacological inhibitor of the extracellular signal-regulated kinase pathway might be therapeutically effective too.

The miRNA expression profiles in normal human or murine NK cells are well documented. ${ }^{3,4}$ When the most abundant 20 miRNAs are compared, only nine are common to malignant NK cells and normal NK cells (Supplemental Figure S2). For example, both normal and malignant NK cells express high levels of miR-21 and miR-142-3p, but miR-494 is found only in malignant NK cells. This finding demonstrates significant alterations during malignant transformation, in part due to the presence of EBV-encoded miRNAs, such as miR-BART20-5p, in malignant NK cells only.

T-bet induces PTEN, TP53, and IFN- $\gamma$, consistent with CHIP-on-CHIP data that T-bet regulates the promoters of PTEN, TP53, and IFNG (Figure 5J). ${ }^{34,35}$ This model does not consider inhibition of TBX21 and IFNG by miR-29, ${ }^{49,50}$ because of the low expression of miR-29 in NNLs (average level, 50) (Supplemental Table S1).

In conclusion, in NNLs, miR-494-3p inhibits the tumor suppressor PTEN with secondary induction of AKT, whereas miR-142-3p inhibits RICTOR with secondary suppression of AKT. We propose a model in which miR-BART20-5p, miR-494-3p, and miR-142-3p cooperate to inhibit a loop, where T-bet induces both PTEN and TP53 to inhibit AKT. This model is consistent with recent molecular data that the AKT pathway is critical in the pathogenesis of $\mathrm{NNL},{ }^{6,8,51}$ suggesting potential targets of therapy through antagomirs to miR494-3p, mimics of miR-142-3p, or pharmacological manipulations of the phosphatidylinositol 3-kinase/AKT/ mTOR pathway. ${ }^{52}$

\section{Acknowledgments}

We thank the Tissue Bank at the National Taiwan University Center for Genomic Medicine for providing technical support.

H.-H.C. constructed plasmids and performed experiments on cell lines, including luciferase assays, chromatin immunoprecipitation, and Western blot analyses; W.-T.H. and L.-W.Y. provided additional technical support; C.-W.L. designed the experiments and wrote the manuscript. 


\section{Supplemental Data}

Supplemental material for this article can be found at http://dx.doi.org/10.1016/j.ajpath.2015.01.025.

\section{References}

1. Lin TC, Liu TY, Hsu SM, Lin CW: The EBV-encoded miRBART20-5p inhibits T-bet with secondary suppression of p53 in invasive nasal NK-cell lymphoma. Am J Pathol 2013, 182: $1865-1875$

2. Huang WT, Lin CW: EBV-encoded miR-BART20-5p and miRBART8 inhibit the IFN $\gamma$-STAT1 pathway associated with disease progression in nasal NK-cell lymphoma. Am J Pathol 2014, 184: $1185-1197$

3. Landgraf P, Rusu M, Sheridan R, Sewer A, Iovino N, Aravin A, et al: A mammalian microRNA expression atlas based on small RNA library sequencing. Cell 2007, 129:1401-1414

4. Fehniger TA, Wylie T, Germino E, Leong JW, Magrini VJ, Koul S, Keppel CR, Schneider SE, Koboldt DC, Sullivan RP, Heinz ME, Crosby SD, Nagarajan R, Ramsingh G, Link DC, Ley TJ, Mardis ER: Next-generation sequencing identifies the natural killer cell microRNA transcriptome. Genome Res 2010, 20:1590-1604

5. Motsch N, Alles J, Imig J, Zhu J, Barth S, Reineke T, Tinguely M, Cogliatti S, Dueck A, Meister G, Renner C, Grässer FA: MicroRNA profiling of Epstein-Barr virus-associated NK/T-cell lymphomas by deep sequencing. PLoS One 2012, 7:e42193

6. Yamanaka Y, Tagawa H, Takahashi N, Watanabe A, Guo YM, Iwamoto K, Yamashita J, Saitoh H, Kameoka Y, Shimizu N, Ichinohasama R, Sawada K: Aberrant overexpression of microRNAs activate AKT signaling via down-regulation of tumor suppressors in natural killer-cell lymphoma/leukemia. Blood 2009, 114:3265-3275

7. Chow C, Liu AY, Chan WS, Lei KI, Chan WY, Lo AW: AKT plays a role in the survival of the tumor cells of extranodal NK/T-cell lymphoma, nasal type. Haematologica 2005, 90:274-275

8. Huang Y, de Leval L, Gaulard P: Molecular underpinning of extranodal NK/T-cell lymphoma. Best Pract Res Clin Haematol 2013, 26 : $57-74$

9. Testa RJ, Tsichlis PN: AKT signaling in normal and malignant cells. Oncogene 2005, 24:7391-7393

10. Yin Y, Shen WH: PTEN: a new guardian of the genome. Oncogene 2008, 27:5443-5453

11. Mayo LD, Donner DB: The PTEN, Mdm2, p53 tumor suppressoroncoprotein network. Trends Biochem Sci 2002, 27:462-467

12. Freeman DJ, Li AG, Wei G, Li HH, Kertesz N, Lesche R, Whale AD, Martinez-Diaz H, Rozengurt N, Cardiff RD, Liu X, Wu H: PTEN tumor suppressor regulates p53 protein levels and activity through phosphatase-dependent and -independent mechanisms. Cancer Cell 2003, 3:117-130

13. Ogawara Y, Kishishita S, Obata T, Isazawa Y, Suzuki T, Tanaka K, Masuyama N, Gotoh Y: Akt enhances Mdm2-mediated ubiquitination and degradation of p53. J Biol Chem 2002, 277:21843-21850

14. Li AG, Piluso LG, Cai X, Wei G, Sellers WR, Liu X: Mechanistic insights into maintenance of high p53 acetylation by PTEN. Mol Cell 2006, 23:575-587

15. Haupt S, Berger M, Goldberg Z, Haupt Y: Apoptosis: the p53 network. J Cell Sci 2003, 116:4077-4085

16. Sarbassov DD, Guertin DA, Ali SM, Sabatini DM: Phosphorylation and regulation of Akt/PKB by the Rictor-mTOR complex. Science 2005, 307:1098-1101

17. Kalaitzidis D, Sykes SM, Wang Z, Punt N, Tang Y, Ragu C, Sinha AU, Lane SW, Souza AL, Clish CB, Anastasiou D, Gilliland DG, Scadden DT, Guertin DA, Armstrong SA: mTOR complex 1 plays critical roles in hematopoiesis and Pten-loss-evoked leukemogenesis. Cell Stem Cell 2012, 11:429-439
18. Hoshii T, Kasada A, Hatakeyama T, Ohtani M, Tadokoro Y, Naka K, Ikenoue $\mathrm{T}$, Ikawa $\mathrm{T}$, Kawamoto $\mathrm{H}$, Fehling $\mathrm{HJ}$, Araki $\mathrm{K}$, Yamamura K, Matsuda S, Hirao A: Loss of mTOR complex 1 induces developmental blockage in early T-lymphopoiesis and eradicates T-cell acute lymphoblastic leukemia cells. Proc Natl Acad Sci U S A 2014, 111:3805-3810

19. Lee K, Nam KT, Cho SH, Gudapati P, Hwang Y, Park DS, Potter R, Chen J, Volanakis E, Boothby M: Vital roles of mTOR complex 2 in Notch-driven thymocyte differentiation and leukemia. J Exp Med 2012, 209:713-728

20. Gupta M, Ansell SM, Novak AJ, Kumar S, Kaufmann SH, Witzig TE: Inhibition of histone deacetylase overcomes rapamycinmediated resistance in diffuse large B-cell lymphoma by inhibiting Akt signaling through mTORC2. Blood 2009, 114:2926-2935

21. Geiss GK, Bumgarner RE, Birditt B, Dahl T, Dowidar N, Dunaway DL, Fell HP, Ferree S, George RD, Grogan T, James JJ, Maysuria M, Mitton JD, Oliveri P, Osborn JL, Peng T, Ratcliffe AL, Webster PJ, Davidson EH, Hood L, Dimitrov K: Direct multiplexed measurement of gene expression with color-coded probe pairs. Nat Biotechnol 2008, 26:317-325

22. Vladislav MA: Multiplexed measurements of gene signatures in different analytes using the NanoString nCounter Assay System. BMC Res Notes 2009, 2:80

23. Kulkarni MM: Digital multiplexed gene expression analysis using the NanoString nCounter System. Curr Protoc Mol Biol 2011, ch 25: Unit25B.10

24. Alexiou P, Maragkakis M, Papadopoulos GL, Simmosis VA, Zhang L, Hatzigeorgiou AG: The DIANA-mirExTra web server: from gene expression data to microRNA function. PLoS One 2010, 5: e9171

25. Krüger J, Rehmsmeier M: RNAhybrid: microRNA target prediction easy, fast and flexible. Nucleic Acids Res 2006, 34(Web Server issue):W451-W454

26. Zhou Y, Feng X, Liu YL, Ye SC, Wang H, Tan WK, Tian T, Qiu YM, Luo HS: Downregulation of miR-126 is associated with colorectal cancer cells proliferation, migration and invasion by targeting IRS-1 via the AKT and ERK1/2 signaling pathways. PLoS One 2013, 8:e81203

27. Liu L, Jiang Y, Zhang H, Greenlee AR, Han Z: Overexpressed miR494 down-regulates PTEN gene expression in cells transformed by anti-benzo(a)pyrene-trans-7,8-dihydrodiol-9, 10-epoxide. Life Sci 2010, 86:192-198

28. Liu Y, Lai L, Chen Q, Song Y, Xu S, Ma F, Wang X, Wang J, Yu H, Cao X, Wang Q: MicroRNA-494 is required for the accumulation and functions of tumor-expanded myeloid-derived suppressor cells via targeting of PTEN. J Immunol 2012, 188:5500-5510

29. Meng F, Henson R, Wehbe-Janek H, Ghoshal K, Jacob ST, Patel T: MicroRNA-21 regulates expression of the PTEN tumor suppressor gene in human hepatocellular cancer. Gastroenterology 2007, 133: 647-658

30. Ma X, Kumar M, Choudhury SN, Becker Buscaglia LE, Barker JR, Kanakamedala K, Liu MF, Li Y: Loss of the miR-21 allele elevates the expression of its target genes and reduces tumorigenesis. Proc Natl Acad Sci U S A 2011, 108:10144-10149

31. Ma L, Chen Z, Erdjument-Bromage H, Tempst P, Pandolfi PP: Phosphorylation and functional inactivation of TSC2 by Erk implications for tuberous sclerosis and cancer pathogenesis. Cell 2005, 121:179-193

32. Hatley ME, Patrick DM, Garcia MR, Richardson JA, BasselDuby R, van Rooij E, Olson EN: Modulation of K-Ras-dependent lung tumorigenesis by MicroRNA-21. Cancer Cell 2010, 18: 282-293

33. Tian Y, Nan Y, Han L, Zhang A, Wang G, Jia Z, Hao J, Pu P, Zhong Y, Kang C: MiR-451 downregulates the PI3K/AKT pathway through CAB39 in human glioma. Int J Oncol 2012, 40:1105-1112

34. Kanhere A, Hertweck A, Bhatia U, Gökmen MR, Perucha E, Jackson I, Lord GM, Jenner RG: T-bet and GATA3 orchestrate Th1 
and Th2 differentiation through lineage-specific targeting of distal regulatory elements. Nat Commun 2012, 3:1268

35. Jenner RG, Townsend MJ, Jackson I, Sun K, Bouwman RD, Young RA, Glimcher LH, Lord GM: The transcription factors T-bet and GATA-3 control alternative pathways of T-cell differentiation through a shared set of target genes. Proc Natl Acad Sci U S A 2009, 106:17876-17881

36. el-Deiry WS, Kern SE, Pietenpol JA, Kinzler KW, Vogelstein B: Definition of a consensus binding site for p53. Nat Genet 1992, 1: 45-49

37. Vereide D, Sugden B: Insights into the evolution of lymphomas induced by Epstein-Barr virus. Adv Cancer Res 2010, 108:1-19

38. Balaga O, Friedman Y, Linial M: Toward a combinatorial nature of microRNA regulation in human cells. Nucleic Acids Res 2012, 40: 9404-9416

39. Dou L, Li J, Zheng D, Li Y, Gao X, Xu C, Gao L, Wang L, Yu L: MicroRNA-142-3p inhibits cell proliferation in human acute lymphoblastic leukemia by targeting the MLL-AF4 oncogene. Mol Biol Rep 2013, 40:6811-6819

40. Lv M, Zhang X, Jia H, Li D, Zhang B, Zhang H, Hong M, Jiang T, Jiang Q, Lu J, Huang X, Huang B: An oncogenic role of miR-142-3p in human T-cell acute lymphoblastic leukemia (T-ALL) by targeting glucocorticoid receptor- $\alpha$ and cAMP/PKA pathways. Leukemia 2012, 26:769-777

41. Swami M: Therapeutics: Akt: a double-edged sword. Nat Rev Cancer 2008, 9:76-77

42. Nogueira V, Park Y, Chen CC, Xu PZ, Chen ML, Tonic I, Unterman T, Hay $\mathrm{N}$ : Akt determines replicative senescence and oxidative or oncogenic premature senescence and sensitizes cells to oxidative stress. Cancer Cell 2008, 14:458-470

43. Mone AP, Huang P, Pelicano H, Cheney CM, Green JM, Tso JY, Johnson AJ, Jefferson S, Lin TS, Byrd JC: Hu1D10 induces apoptosis concurrent with activation of the AKT survival pathway in human chronic lymphocytic leukemia cells. Blood 2004, 103:1846-1854
44. Pfeifer M, Grau M, Lenze D, Wenzel SS, Wolf A, Wollert-Wulf B, Dietze K, Nogai H, Storek B, Madle H, Dörken B, Janz M, Dirnhofer S, Lenz P, Hummel M, Tzankov A, Lenz G: PTEN loss defines a PI3K/AKT pathway-dependent germinal center subtype of diffuse large B-cell lymphoma. Proc Natl Acad Sci U S A 2013, 110: $12420-12425$

45. Pfeifer M, Lenz G: PI3K/AKT addiction in subsets of diffuse large Bcell lymphoma. Cell Cycle 2013, 12:3347-3348

46. Gu L, Song G, Chen L, Nie Z, He B, Pan Y, Xu Y, Li R, Gao T, Cho WC, Wang S: Inhibition of miR-21 induces biological and behavioral alterations in diffuse large B-cell lymphoma. Acta Haematol 2013, 130:87-94

47. Bai H, Wei J, Deng C, Yang X, Wang C, Xu R: MicroRNA-21 regulates the sensitivity of diffuse large B-cell lymphoma cells to the CHOP chemotherapy regimen. Int J Hematol 2013, 97:223-231

48. Romano G, Acunzo M, Garofalo M, Di Leva G, Cascione L, Zanca C, Bolon B, Condorelli G, Croce CM: MiR-494 is regulated by ERK1/2 and modulates TRAIL-induced apoptosis in non-small-cell lung cancer through BIM down-regulation. Proc Natl Acad Sci U S A 2012, 109:16570-16575

49. Steiner DF, Thomas MF, Hu JK, Yang Z, Babiarz JE, Allen CD, Matloubian M, Blelloch R, Ansel KM: MicroRNA-29 regulates T-box transcription factors and interferon- $\gamma$ production in helper $\mathrm{T}$ cells. Immunity 2011, 35:169-181

50. Ma F, Xu S, Liu X, Zhang Q, Xu X, Liu M, Hua M, Li N, Yao H, Cao X: The microRNA miR-29 controls innate and adaptive immune responses to intracellular bacterial infection by targeting interferon- $\gamma$. Nat Immunol 2011, 12:861-869

51. Ng SB, Yan J, Huang G, Selvarajan V, Tay JL, Lin B, Bi C, Tan J, Kwong YL, Shimizu N, Aozasa K, Chng WJ: Dysregulated microRNAs affect pathways and targets of biologic relevance in nasal-type natural killer/T-cell lymphoma. Blood 2011, 118:4919-4929

52. Westin JR: Status of PI3K/Akt/mTOR pathway inhibitors in lymphoma. Clin Lymphoma Myeloma Leuk 2014, 14:335-342 